\title{
Ampirik Mod Ayrıştırması ve Welch Yöntemini Kullanarak Dört Sınıflı Motor Hayali EEG Sinyallerinin Derin Öğrenme ile Sinıflandırılması
}

\author{
Mustafa Tosun ${ }^{1 *}$, Osman Çetin ${ }^{2}$ \\ ${ }^{1 *}$ Dumlupınar Üniversitesi, Simav Teknoloji Fak., Elektrik Elektronik Müh. Bölümü, Kütahya, Türkiye (ORCID: 0000-0001-7167-4561), mustafa.tosun@dpu.edu.tr \\ 2 Dumlupınar Üniversitesi, Simav Teknoloji Fak., Elektrik Elektronik Müh. Bölümü, Kütahya, Türkiye (ORCID: 0000-0001-8988-5025), osman.cetin@dpu.edu.tr
}

(3rd International Congress on Human-Computer Interaction, Optimization and Robotic Applications June 11-13, 2021)

(DOI: 10.31590 /ejosat.948099)

ATIF/REFERENCE: Tosun, M. \& Çetin, O. (2021). Ampirik Mod Ayrıştırması ve Welch Yöntemini Kullanarak Dört Sınıflı Motor Hayali EEG Sinyallerinin Derin Öğrenme ile Sınıflandırılması. Avrupa Bilim ve Teknoloji Dergisi, (26), 284-288.

$\ddot{O} \mathbf{z}$

Elektroensefalogram (EEG) tabanlı beyin-bilgisayar arayüzü (BBA) uygulamalarında, kişilerin ilgili uzuv hareketlerini hayal etmesiyle elde edilen motor hayali (MI) sinyallerinden özellik çıkarmak ve bunları sınıflandırmak oldukça önemli bir konudur. MI-EEG sinyalleriyle ilgili yapılan çalışmalarda, birçok farklı özellik çıkarma yöntemleri ve sınıflandırma algoritmaları kullanılmıştır. Fakat bu sinyallerde sınıf sayısı arttıkça elde edilen sınıflandırma başarıları arasında belirgin farklar gözlemlenmiştir. Önerilen yöntemde, sinyallerin güç spektral yoğunluğu (PSD) bilgilerini içeren özellik çıkarma yöntemi sunulmuştur. Ham EEG verilerine ampirik mod ayrıştırması (EMD) uygulanarak farklı frekans seviyelerindeki sinyaller elde edilmiştir. Bu sinyallerin PSD değerleri welch yöntemi kullanılarak hesaplanmıştır. Elde edilen PSD değerleri bir öznitelik vektöründe birleştirilmiştir. Oluşturulan öznitelik vektörlerini kullanarak, popüler bir derin öğrenme algoritması olan uzun-kısa dönem hafıza (LSTM) ağı eğitilmişstir. Eğitim sonucunda elde edilen test başarılarının, kişiler ve kanallar bazındaki karşılaştırmaları detaylı olarak yapılmıştır. Karşılaştırma sonucunda kafa derisinin merkez noktasında bulunan kanalların, diğer kanallara göre daha başarılı oldukları görülmüştür.

Anahtar Kelimeler: Ampirik Mod Ayrıştırması, Beyin-Bilgisayar Arayüzü, Uzun-Kısa Dönem Hafıza, Welch Yöntemi.

\section{Classification of Four-Class Motor Imaginary EEG Signals with Deep Learning Using Empirical Mode Decomposition and Welch Method}

\begin{abstract}
In electroencephalogram (EEG) based brain-computer interface (BCI) applications, it is very important to extract features from motor imagery (MI) signals obtained by imagining related limb movements and to classify them. Many different feature extraction methods and classification algorithms have been used in studies on MI-EEG signals. However, significant differences have been observed between the classification accuracies obtained as the number of classes increased in these signals. In the proposed method, feature extraction method including power spectral density (PSD) information of signals is presented. By applying empirical mode decomposition (EMD) to the raw EEG data, signals at different frequency levels were obtained. The PSD values of these signals were calculated using the welch method. The PSD values obtained were combined in a feature vector. Using the generated feature vectors, the long-short term memory (LSTM) network, a popular deep learning algorithm, was trained. The comparisons of the test accuracies obtained as a result of the training on the basis of individuals and channels were made in detail. As a result of the comparison, it was observed that the channels at the center of the scalp are more successful than the other channels.
\end{abstract}

Keywords: Empirical Mode Decomposition, Brain-Computer Interface, Long-Short Term Memory, Welch Method.

* Sorumlu Yazar: Dumlupınar Üniversitesi, Simav Teknoloji Fakültesi, Elektrik Elektronik Müh. Bölümü, Kütahya, Türkiye (ORCID: 0000-0001-7167-4561) mustafa.tosun@dpu.edu.tr 


\section{Giriş}

BBA, kişilerin herhangi bir kas gücü gerektirmeden, beyinden alınan biyoelektriksel sinyaller ile harici elektronik cihazlar arasında iletişim kurmalarını sağlayan sistemlerdir. $\mathrm{Bu}$ sistemler; biyomedikal uygulamalarda, motor fonksiyonlarını yerine getiremeyen felçli hastaların tekerlekli sandalye ve robot kol gibi cihazları kontrol etmelerinde, araba kullanma, drone kontrolü gibi çeşitli uygulamalar için kullanılır [1]. Beyin aktivitelerinin ölçülmesinde, EEG, elektrokortigografi (ECoG), fonksiyonel manyetik rezonans görüntüleme (fMRI), magnetoensefalografi (MEG), fonksiyonel yakın infrared spektroskopisi (fNIRS) gibi çeşitli yöntemler mevcuttur. Bu yöntemler arasında düşük maliyeti, taşınabilirliliği ve sinyal elde etmenin kolaylığı nedeniyle çoğunlukla EEG sinyalleri tercih edilir [2]. BBA ile ilgi yapılan çalışmalarda, motor hayali, kararlı durum görsel uyarılmış potansiyelleri (SSVEP) ve P300 gibi çeşitli EEG sinyalleri kullanılmıştır [3]. Bu sinyaller arasında motor hayali sinyalleri, kişide herhangi bir dürtüye gerek olmadan kendiliğinden üretilebildiği için en yaygın kullanılmakta olan sinyallerdir [4]. Motor hayali, bir kişi el veya bacak gibi uzuvlarının hareketini hayal ettiğinde ve hayalini bitirdiğinde, elektrotlardan kaydedilen beynin elektriksel aktivitesidir [5].

BBA uygulamalarında gerekli özniteliklerin çıkartılması ve bunların sınıflandırılması oldukça önemli bir konudur. Çünkü EEG sinyalleri beyindeki milyarlarca nöronun etkileşimi sonucunda elde edilen aperiyodik ve non-lineer sinyallerdir [6]. Aynı zamanda EEG sinyalleri, iç ve dış etkenlerden kaynaklanan gürültülere karşı oldukça hassastırlar [7]. EEG sinyallerinden özellik çıkarmada; ortak uzamsal örüntüler (CSP) [8], ayrık dalgacık dönüşümü (DWT) [9], hızlı Fourier dönüşümü (FFT) [10], kısa süreli fourier dönüşümü (STFT) [11] ve Hilbert-Huang dönüşümü (HHT) [12] gibi birçok farklı yöntem kullanılmıştır. Öznitelikleri sınıflandırmada; destek vektör makineleri (SVM) [13], doğrusal diskrimant analizi (LDA) [14], en yakın komşuluk ilişkisi (k-NN) [15] gibi çeşitli yöntemler kullanılmıştır. Fakat makine öğrenmesi tekniklerini kullanarak beyin sinyallerinin modellenmesi oldukça zordur. Geleneksel makine öğrenmesi yöntemleriyle sınıflandırmada yüksek doğruluklar elde edilememiştir.

Son yıllarda, araştırmacılar tarafından derin öğrenme yöntemlerine ilgi artmıştır. Derin öğrenme yöntemleri, konuşma ve görüntü tanıma gibi alanlarda yaygın olarak başarıyla kullanılmıştır [16]. Bununla birlikte, MI-EEG uygulamalarında derin öğrenme yöntemlerinin kullanımı, konuşma ve görüntü tanıma gibi alanlara göre kısıtlıdır ve daha fazla araştırmaya ihtiyaç vardır [17]. Derin öğrenme algoritmaları kullanılarak yapılan sınıflandırmalarda elde edilen başarı, eğitim verilerinin miktarına bağlıdır. Özellikle, küçük veri kümeleriyle derin öğrenme modelleri eğitilmesi oldukça zordur. Derin öğrenme algoritmalarının öğrenme stratejilerinde çok büyük eğitim verileri gereklidir [18]. Bu nedenle, çalışmada kullanılan mevcut veri setinin tamamı, sinıflandırıcılara uygulanmadan önce 0.9 ve 1.1 [19] ile çarpılarak arttırılmıştır.

Önerilen yöntemde, veri setinde bulunan toplamda 9 kişiye ait 22 farklı kanalın sınıflandırma başarılarına olan etkileri detaylı olarak incelenmiştir. Kanallara ait ham EEG verilerine Ampirik Mod Ayrıştırması uygulanarak farklı frekans seviyelerindeki alt sinyaller elde edilmiştir. Ayrıca, gürültü olarak nitelendirilebilecek en yüksek frekanslı bileşen atılmıştır. Ayrıştırılan sinyallerin PSD değerleri, Welch yöntemi uygulanarak hesaplanmıştır. Ayrıştırılan sinyallerden elde edilen PSD değerlerinin belirleyici olan kısımları bir öznitelik vektöründe birleştirilmiştir. Bu öznitelikler, LSTM derin ağının girişine uygulanmıştır. Eğitim sonucunda elde edilen test başarıları, kişiler ve kanallar bazında olmak üzere tablo halinde gösterilmiştir.

\section{Materyal ve Metot}

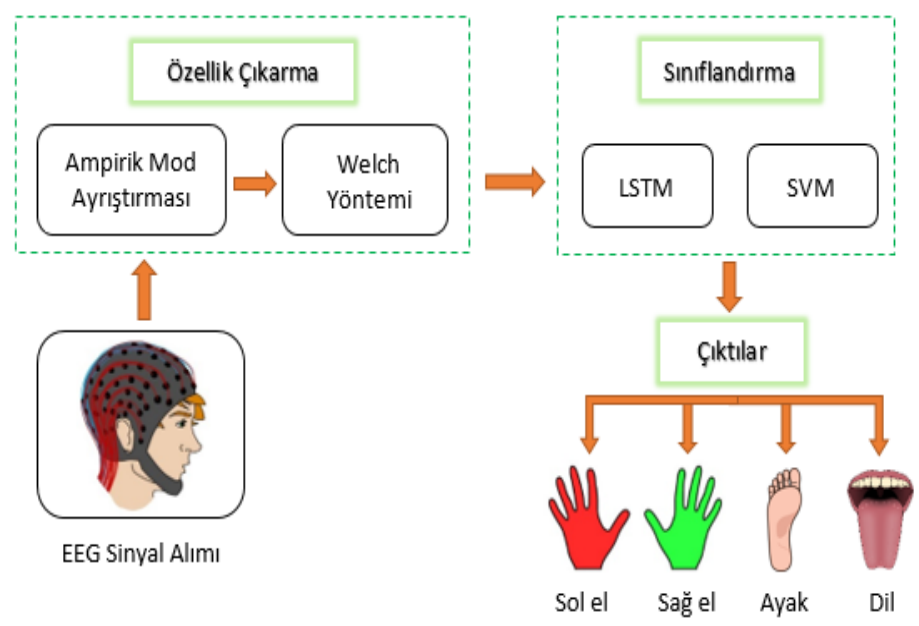

Şekil 1. Önerilen yöntemin blok diyagramı

Önerilen yöntemin blok diyagramı Şekil 1' de gösterilmiştir. Önerilen yöntemde, özellik çıkarma yöntemi olarak EMD algoritmasıyla birlikte Welch yöntemi kullanılmıştır. 22 kanala ait EEG verilerine EMD algoritması uygulanarak, her bir kanal için 4 farklı frekans bandı aralığına sahip (IMF-2, İMF-3, İMF-4 ve İMF-5) sinyalleri elde edilmiştir. İMF-1 sinyali, yüksek frekanslı gürültü bileşenlerini içerdiğinden bu çalışmada kullanılmamıştır. Ardından, Welch yöntemi uygulanarak bu sinyallerin PSD değerleri hesaplanmıştır. Elde edilen PSD değerlerinin belirleyici olan kısımları bir öznitelik vektöründe birleştirilmiştir. EMD ve Welch yöntemlerini birlikte kullanarak elde edilen öznitelik vektörleri, LSTM derin ağıyla sınıflandırılmıştır.

\subsection{EEG Veri Kaydı}

Bu çalışmada BCI Competition IV-2a veri seti kullanılmıştır [20]. Çalışılan veri seti, 9 farklı kişiden alınan sol el, sağ el, ayak ve dil olmak üzere 4 farklı sınıfa ait motor hayali görevlerinden oluşmaktadır. Denekler bilgisayar ekranının önüne oturtularak, her deneme $\mathrm{t}=0 \mathrm{sn}$ ' de uyartım sesiyle birlikte başlamıştır ve siyah ekranda çarpı işareti belirmiştir. İki saniye sonra $\left(\mathrm{t}=2 \mathrm{sn}{ }^{\prime}\right.$ de) sola, sağa, aşağıya ve yukarıya dönük, dört sınıftan birine karşılık gelen bir ok işareti belirmiştir. Daha sonra, deneklere ok işaretine karşılık gelen sınıfın hayali hareketini 3. saniye ile 6 . saniye arasında yapmaları istenmiştir. Elektrotlar, uluslararası 1020 sistemine göre kafa derisi üzerine yerleştirilmiştir. Zamanlama şeması Şekil 2' de gösterilmiştir. 


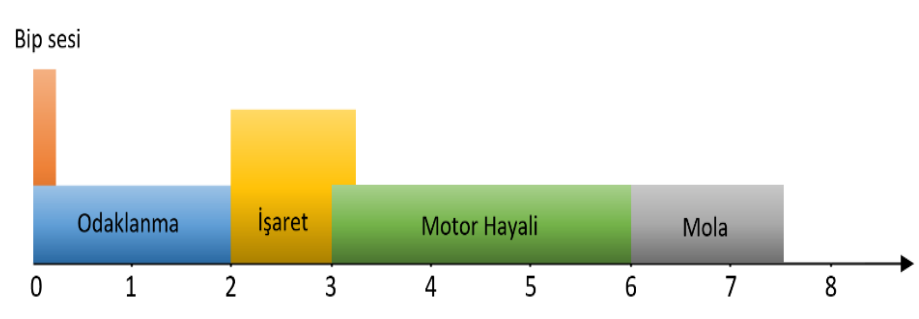

Şekil 2. EEG kaydının zamanlama şeması

\subsection{Ampirik Mod Ayrıştırması (EMD)}

EMD yöntemi, herhangi bir gerçek değerli giriş sinyalinin farklı salınımlar yapan basit alt sinyallerin toplamından oluştuğu prensibine dayanmaktadır. Bu alt sinyallerin her birisi içsel mod fonksiyonları (IMF) olarak isimlendirilir. Bir sinyalden IMF çıkarma işlemi "eleme" olarak adlandırılır. İlk olarak, giriş sinyalindeki tüm yerel extremum noktaları (minimumlar ve maksimumlar) belirlenir. Daha sonra tüm yerel maksimum noktaları üst zarf, tüm yerel minimum noktaları ise alt zarf olacak şekilde birleştirilir. Üst ve alt zarfların ortalaması $\mathrm{m} 1$ olarak adlandırılır. Giriş sinyali $x(t)$ ve $m_{1}$ sinyalinin farkı $h_{1}$ olarak adlandırılır ve 1 no' lu bağıntıyla gösterilir [21].

$h_{1}=x(t)-m_{1}$

Eleme işlemi, üst ve alt zarflar simetrik yapıda oluncaya kadar devam eder. Bir sonraki aşamada h1 sinyali proto-iMF olarak kabul edilir ve yeni giriş verisi olarak ele alınır. Diğer İMF, bu sinyalden elde edilir. Üst ve alt zarfların ortalaması $m_{11}$ olarak adlandırılır. Yeni giriş verisi $h_{1}$ ve $m_{11}$ arasındaki fark $h_{11}$ olarak adlandırılır ve 2 no' lu bağıntıyla gösterilir [21].

$h_{11}=h_{1}-m_{11}$

$\mathrm{k}$ kez tekrarlanan eleme işleminden sonra, yerel zarfların simetri koşulu karşılanır. İlk İMF bileşeni $c_{1 k}$ olarak adlandırılır ve 3 no' lu bağıntıyla gösterilir. Benzer mantıkla, giriş sinyalinin eğer varsa diğer İMF'leri, bir önceki İMF üzerinden elde edilir [21].

$c_{1 k}=h_{1(k-1)}-m_{1 k}$

\subsection{Welch Yöntemi}

Welch yönteminde, kesikli giriş sinyali belirli uzunluktaki dilimlere bölünür. Her bir dilimin, isteğe bağlı olarak seçilmiş bir pencere fonksiyonuyla çarpıldıktan sonra yumuşatılmış periodogramları hesaplanır. Ardından, i. yumuşatılmış periodogram ifadesi Eşitlik 4' deki gibi gösterilir [22].

$\hat{P}_{i}(f)=\frac{1}{M} \frac{1}{H}\left[\sum_{n=0}^{M-1} w[n] x_{i}[n] e^{-j 2 \pi f n}\right]^{2}$

Eşitlik 4' deki w[n] ifadesi, isteğe bağl1 olarak seçilen herhangi bir pencere fonksiyodur. Genellikle "hamming" penceresi tercih edilir. M ifadesi, dilim uzunluğudur ve her bir dilim için eşittir. $\mathrm{H}$ ifadesi pencere fonksiyonunun normalize edilmiş halidir ve Eşitlik 5' te gösterilmiştir [22].

$H=\frac{1}{M} \sum_{n=0}^{M-1} w[n]^{2}$
Son olarak, Welch' in güç spektral tahmini, yumuşatılmış periodogramların ortalaması alınarak elde edilir ve Eşitlik 6' daki gibi gösterilir [22].

$$
\widehat{P}_{\text {welch }}(f)=\frac{1}{s} \sum_{i=1}^{S} \hat{P}_{i}(f)
$$

\subsection{Uzun-Kısa Dönem Hafıza (LSTM)}

LSTM ağları, yinelenen sinir ağlarını (RNN) özel bir türü olup, kapılar aracılı̆ııla hücre durumlarına bilgi ekleme veya kaldırma yeteneğine sahiptir. LSTM derin öğrenme ünitesi 3 kapıdan oluşmaktadır. Bu kapılar sırasıyla unutma kapısı, giriş kapısı ve çıkış kapısıdır. Unutma kapısı sigmoid katmandan oluşur ve hangi bilgilerin atılacağına karar verir. Giriş kapısı, sigmoid ve hiperbolik tanjant katmanlarından oluşmaktadır. Sigmoid katmanı, hangi bilgilerin güncelleneceğine karar verir. Tanjant katmanı güncellenmiş yeni değerlerin vektörünü oluşturur. Çıkış kapısı, sigmoid ve hiperbolik tanjant katmanlarından oluşmaktadır. Öncelikle, güncellenmiş hücre durumu bilgileri hiperbolik tanjant katmanından geçirilir. Ardından, mevcut giriş bilgileri ile bir önceki hücrenin çıktısı sigmoid katmanından geçirilir. Son olarak, her iki katmandan gelen bilgiler çarpılarak hücre çıktısı ifadesi elde edilir [2]. Şekil 3 ’te LSTM hücresi gösterilmiştir.

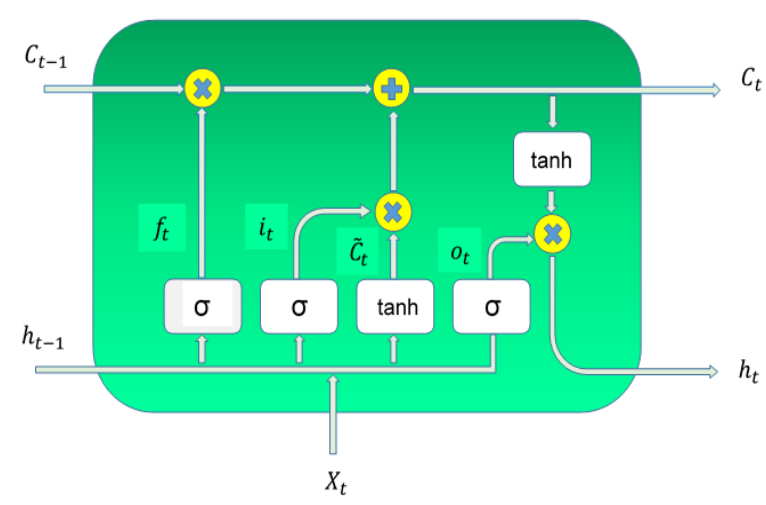

Şekil 3. LSTM hücresinin yapısl

\section{Araştırma Sonuçları ve Tartışma}

$250 \mathrm{hz}$ örnekleme frekansıyla örneklenen EEG sinyalleri, farklı frekanslardaki 4 adet İMF' ye ayrıştırılmıştır. IMF' lerin anlık frekans bilgisinden yola çıkarak elde edilen İMF-5, İMF-4, İMF-3 ve İMF-2 sinyallerinin sirasiyla delta, teta, alfa ve beta bandlarına yaklaşık olarak karşılık geldiği söylenebilir. Bandlara ait PSD değerleri, Welch yöntemi kullanılarak hesaplanmıştır. Welch yönteminde, pencere uzunluğu parametresi veri boyutunun $1 / 3$ ' ü kadar, noverlap parametresi ise pencere uzunluğunun $1 / 2$ ' si kadar seçilmiştir. Ayrıca pencere fonksiyonu olarak Hamming penceresi kullanılmıştır. Nyquist frekansı, $125 \mathrm{hz}$ olduğundan elde edilen grafikler frekans değerleri 125 hz' ye kadardır. Yani, her bir band sinyali için 0' dan 125 hz' ye kadar PSD değeri mevcuttur. Fakat bu PSD değerleri ilgili band aralıklarının dışında çok kü̧̈ük değerlere sahiptir. Bu yüzden sol el, sağ el, ayak ve dil sınıfı için 4 banda ait PSD değerlerinin, sadece ilgili frekans bandı aralıklarındaki kısımları bir öznitelik vektöründe birleştirilmek üzere kullanılmıştır. Delta (0-4 hz), Teta (4-8 hz), Alfa (8-16 hz) ve Beta (16-32 hz) bandlarından elde edilen PSD değerlerinin alt alta eklenmesiyle öznitelik vektörleri oluşturulmuştur. Öznitelik vektörü; delta bandından 12, teta bandından 12, alfa bandından 24 ve beta bandından 48 adet olmak üzere toplamda 96 adet PSD 
değerinden oluşmaktadır. Sonuç olarak, kanal bazında 750 elemandan oluşan EEG verileri, 96 elemanlı PSD değerleriyle temsil edilmiştir. Şekil 4'te sol el, sağ el ayak ve dil sınıflarına ait PSD grafikleri gösterilmiştir.

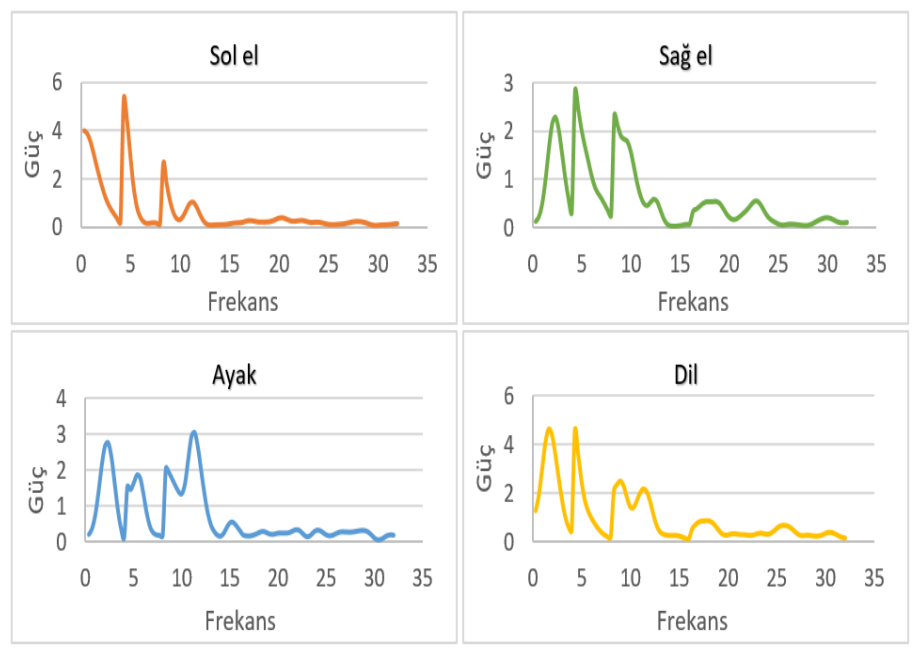

Şekil 4. Band güçlerinin birleştirilmesiyle elde edilen PSD grafikleri

Derin öğrenme algoritmalarının öğrenme stratejilerinde, eğitimleri için çok sayıda örnek gerektiğinden, elde edilen öznitelik vektörleri $\% 90$ ve $\% 110$ faktörleriyle çarpılmıştır. Böylece, öznitelik vektörlerinin sayıları üç kat arttırılmıştır. Kullanılan yöntemlerde kanal bazında çalışıldığından, toplamda 22 kanalın her biri için aynı sayıda veri seti mevcuttur. Her bir kişi için tüm sınıflara ait veriler, 411 adet öznitelik vektöründen oluşmaktadır. Her bir öznitelik vektörü 96 adet PSD değeri içermektedir. Bir kişiye ait sol el, sağ el, ayak ve dil sınıfları için toplamda 1644 adet öznitelik vektörü mevcuttur. LSTM derin ağında, bu öznitelik vektörlerinin \%70' lik kısmı eğitim, \%15' lik kısmı validation ve diğer $\% 15$ ' lik kısmı test için kullanılmıştır. Tablo 1' de, EMD+Welch yöntemiyle oluşturulan öznitelik vektörlerinin, sınıflandırma işleminde kullanılmak üzere kişiler ve sınıflar bazındaki sayıları detaylı olarak gösterilmiştir.

Tablo 1. Sinıflandırmada kullanılan veri seti’ nin dağılımı

\begin{tabular}{l|cccc|ccc}
\hline \hline \multirow{2}{*}{ Kişi } & \multicolumn{4}{|c|}{ Sinıf } & \multicolumn{3}{c}{ Dağılım } \\
& Sol el & Sağ el & Ayak & Dil & Ĕgitim & Val. & Test \\
\cline { 2 - 8 } S1 & 411 & 411 & 411 & 411 & 1148 & 244 & 244 \\
S2 & 411 & 411 & 411 & 411 & 1148 & 244 & 244 \\
S3 & 411 & 411 & 411 & 411 & 1148 & 244 & 244 \\
S4 & 411 & 411 & 411 & 411 & 1148 & 244 & 244 \\
S5 & 411 & 411 & 411 & 411 & 1148 & 244 & 244 \\
S6 & 411 & 411 & 411 & 411 & 1148 & 244 & 244 \\
S7 & 411 & 411 & 411 & 411 & 1148 & 244 & 244 \\
S8 & 411 & 411 & 411 & 411 & 1148 & 244 & 244 \\
S9 & 411 & 411 & 411 & 411 & 1148 & 244 & 244 \\
\hline \hline
\end{tabular}

EMD+Welch yöntemiyle oluşturulan özniteliklerin, LSTM derin ağıyla sınıflandırılması sonucunda elde edilen test başarıları, kişiler ve kanallar bazında olmak üzere Tablo 2' de gösterilmiştir. Kişiler bazında en yüksek başarı, 22 kanalın başarılarının ortalaması alınarak hesaplanmıştır ve 3. kişide $\% 86,85$ olarak eldeedilmiştir. Bu kişiye en yakın başarı ise
$\% 86,47^{\prime}$ lik başarıyla 9 . kişi olmuştur. Kişiler bazında en düşük başarı ise 4 . kişide \%82,62 olarak elde edilmiştir. Kanallar bazında en yüksek başarı, 9 kişiye ait başarıların ortalaması alınarak hesaplanmıştır ve $\mathrm{FCz}$ kanalında \%89,35 olarak elde edilmiştir. Bu kanala en yakın başarı ise \%88,75' lik başarıyla $\mathrm{Cz}$ kanalı olmuştur. Kanallar bazında en düşük başarı ise C6 kanalında \%81,49 olarak elde edilmiştir.

Tablo 2. Elde edilen test başarıları

\begin{tabular}{cccccccccc}
\hline \hline & \multicolumn{7}{c}{ Kaşiler } \\
Kanal & S1 & S2 & S3 & S4 & S5 & S6 & S7 & S8 & S9 \\
\cline { 2 - 9 } & 92,21 & 90,98 & 88,93 & 76,64 & 89,92 & 87,90 & 90,32 & 87,50 & 85,08 \\
FC3 & 85,66 & 81,97 & 84,84 & 73,77 & 90,32 & 88,71 & 87,90 & 91,13 & 86,69 \\
FC1 & 90,57 & 90,92 & 89,34 & 84,02 & 77,42 & 76,61 & 81,45 & 86,69 & 86,65 \\
FCz & 88,52 & 90,16 & 87,70 & 88,52 & 87,50 & 89,52 & 89,52 & 90,73 & $\mathbf{9 1 , 9 4}$ \\
FC2 & 84,84 & 75,41 & 87,65 & 77,05 & 87,45 & 88,31 & 90,32 & 83,06 & 87,50 \\
FC4 & 86,89 & 84,84 & 88,52 & 89,75 & 84,68 & 85,86 & $\mathbf{9 0 , 7 3}$ & 80,65 & 88,71 \\
C5 & 74,18 & 84,43 & 87,68 & 79,92 & 89,11 & 87,50 & 73,39 & 84,68 & 72,98 \\
C3 & $\mathbf{9 4 , 2 6}$ & 86,07 & 88,11 & 76,23 & 80,65 & 77,82 & 73,79 & $\mathbf{9 2 , 3 4}$ & 90,32 \\
C1 & 89,75 & 86,48 & 91,39 & $\mathbf{9 1 , 8 0}$ & 86,69 & 88,31 & 80,65 & 79,44 & 89,92 \\
Cz & 85,25 & 91,80 & 91,32 & 81,56 & $\mathbf{9 1 , 5 3}$ & 89,11 & 89,92 & 88,71 & 89,52 \\
C2 & 93,03 & 89,34 & 90,16 & 86,07 & 84,27 & $\mathbf{9 1 , 6 2}$ & 85,08 & 87,90 & 89,88 \\
C4 & 90,16 & 77,05 & 84,81 & 76,64 & 85,89 & 89,92 & 84,27 & 83,87 & 83,87 \\
C6 & 76,64 & 88,11 & 77,46 & 78,28 & 82,66 & 79,03 & 80,65 & 87,10 & 83,47 \\
CP3 & 88,52 & 89,75 & 86,48 & 82,38 & 78,23 & 74,60 & 83,06 & 91,94 & 87,90 \\
CP1 & 77,46 & 86,48 & 82,79 & 84,80 & 91,13 & 83,06 & 89,92 & 88,71 & 89,52 \\
CPz & 86,07 & 90,16 & 84,02 & 84,02 & 90,32 & 84,27 & 85,08 & 89,52 & 81,85 \\
CP2 & 90,57 & 77,87 & 77,42 & 81,56 & 89,52 & 91,13 & 89,52 & 87,50 & 86,29 \\
CP4 & 90,53 & $\mathbf{9 2 , 2 1}$ & 88,07 & 82,38 & 79,03 & 77,42 & 85,05 & 74,19 & 89,11 \\
P1 & 81,56 & 73,36 & 90,16 & 88,52 & 88,71 & 83,06 & 78,23 & 90,32 & 91,13 \\
Pz & 73,36 & 91,80 & 82,85 & 86,07 & 89,52 & 80,65 & 81,45 & 89,92 & 80,24 \\
P2 & 84,84 & 77,82 & $\mathbf{9 1 , 7 5}$ & 87,70 & 91,50 & 86,69 & 80,24 & 77,42 & 84,27 \\
POz & 90,50 & 82,79 & 89,34 & 79,92 & 76,61 & 82,66 & 82,26 & 87,10 & 85,48 \\
\hline \hline & & & & & & & & &
\end{tabular}

\section{Sonuç}

Literatürdeki motor hayali sinyallerinin sınıflandırılmasıyla ilgili yapılan çalışmalarda, derin öğrenme algoritmalarının klasik makine öğrenmesi yöntemlerinden daha başarılı oldukları görülmüştür. Ayrıca çok fazla eğitim verisinin olduğu durumlarda, klasik makine öğrenmesi yöntemleriyle yapılan eğitimin süresi oldukça uzundur ve hesaplama verimliliği düşüktür. Derin öğrenme algoritmalarının öğrenme stratejilerinde ise çok fazla eğitim verisi bulunması gerekir. Derin öğrenme algoritmaları, eğitimleri boyunca ne kadar çok fazla veriyle eğitilirse elde edilen sınıflandırma başarıları da o derece yüksek olur. Bir BBA sisteminin anlık tepkilere net cevaplar verebilmesi çok fazla veriyle yapılan iyi bir eğitim sürecine bağlıdır. $\mathrm{Bu}$ sebeple, herhangi bir BBA sisteminde uygulanabilirlik açısından derin öğrenme algoritmaları kullanmak daha doğrudur.

\section{Kaynakça}

1. Amin, S. U., Alsulaiman, M., Muhammad, G., Mekhtiche, M. A., Hossain, M. S. (2019). Deep Learning for EEG motor imagery classification based on multi-layer CNNs feature fusion. Future Generation computer systems, 101, 542-554. 
2. Wang, L., Zhang, X., Zhong, X., Zhang, Y. (2013). Analysis and classification of speech imagery EEG for BCI. Biomedical signal processing and control, 8 (6), 901908. Congress on Computer Science and Engineering (APWC on CSE) (s. 34-39). IEEE.

3. Aydemir, O., \& Kayikcioglu, T. (2014). Decision tree structure based classification of EEG signals recorded during two dimensional cursor movement imagery. Journal of neuroscience methods, 229, 68-75.

4. Li, F., He, F., Wang, F., Zhang, D., Xia, Y., Li, X. (2020). A Novel Simplified Convolutional Neural Network Classification Algorithm of Motor Imagery EEG Signals Based on Deep Learning. Applied Sciences, 10 (5), 1605.

5. Wang, T., Deng, J., He, B. (2004). Classifying EEG-based motor imagery tasks by means of time-frequency synthesized spatial patterns. Clinical Neurophysiology, 115 (12), 27442753.

6. Kam, T. E., Suk, H. I., Lee, S. W. (2013). Non-homogeneous spatial filter optimizationfor Electroencephalogram (EEG)based motor imagery classification. Neurocomputing, 108, 58-68.

7. Tosun, M., \& Kasım, Ö. (2020). Novel eye-blink artefact detection algorithm from raw EEG signals using FCN-based semantic segmentation method. IET Signal Processing, 14(8), 489-494.

8. Selim, S., Tantawi, M. M., Shedeed, H. A., Badr, A. (2018). A CSP\AM-BA-SVM Approach for Motor Imagery BCI System. IEEE Access, 6, 49192-49208.

9. Kumar, S. U., Inbarani, H. H. (2017). PSO-based feature selection and neighborhood rough set-based classification for BCI multiclass motor imagery task. Neural Computing and Applications, 28 (11), 3239-3258.

10. Rodríguez-Bermúdez, G., García-Laencina, P. J. (2012). Automatic and adaptive classification of electroencephalographic signals for brain computer interfaces. Journal of medical systems, 36 (1), 51-63.

11. Ge, S., Wang, R., Yu, D. (2014). Classification of four-class motor imagery employing single-channel electroencephalography. PloS one, 9 (6), e98019.

12. Yuyi, Z., Surui, L., Lijuan, S., Zhenxin, L., Bingchao, D. (2017). Motor imagery eeg discrimination using hilbert-huang entropy.

13. Kim, C., Sun, J., Liu, D., Wang, Q., Paek, S. (2018). An effective feature extraction method by power spectral density of EEG signal for 2-class motor imagery-based BCI. Medical biological engineering computing, 56 (9), 1645-1658.

14. Xie, X., Yu, Z. L., Lu, H., Gu, Z., \& Li, Y. (2016). Motor imagery classification based on bilinear sub-manifold learning of symmetric positivedefinite matrices. IEEE Transactions on Neural Systems and Rehabilitation Engineering, 25(6), 504516.

15. Kevric, J., Subasi, A. (2017). Comparison of signal decomposition methods in classification of EEG signals for motor-imagery BCI system. Biomedical Signal Processing and Control, 31, 398-406.

16. Amin, S. U., Alsulaiman, M., Muhammad, G., Bencherif, M. A., Hossain, M. S. (2019). Multilevel weighted feature fusion using convolutional neural networks for EEG motor imagery classification. IEEE Access, 7, 18940-18950.

17. Jirayucharoensak, S., S. Pan-Ngum ve P. Israsena, (2014). "EEG-Based Emotion Recognition Using Deep Learning Network with Principal Component Based Covariate Shift Adaptation," The Scientific World Journal, C. s. 10.

18. Zhang, Z., Duan, F., Sole-Casals, J., Dinares-Ferran, J., Cichocki, A., Yang, Z., Sun, Z. (2019). A novel deep learning approach with data augmentation to classify motor imagery signals. IEEE Access, 7, 15945-15954.

19. Sakai, A., Minoda, Y., \& Morikawa, K. Data augmentation methods for machine-learning-based classification of biosignals. In 2017 10th Biomedical Engineering International Conference (BMEiCON) (pp. 1-4). IEEE.

20. Brunner, C., Leeb, R., Müller-Putz, G., Schlögl, A., Pfurtscheller, G. (2008). BCI Competition 2008-Graz data set A. Institute for Knowledge Discovery (Laboratory of BrainComputer Interfaces), Graz University of Technology, 16.

21. Pigorini, A., Casali, A. G., Casarotto, S., Ferrarelli, F., Baselli, G., Mariotti, M., Rosanova, M. (2011). Time-frequency spectral analysis of TMS-evoked EEG oscillations by means of Hilbert-Huang transform. Journal of neuroscience methods, 198 (2), 236-245.

22. Alkan, A., Kiymik, M. K. (2006). Comparison of AR and Welch methods in epileptic seizure detection. Journal of Medical Systems, 30 (6), 413-419.

23. Alhagry, S., Fahmy, A. A., El-Khoribi, R. A. (2017). Emotion recognition based on EEG using LSTM recurrent neural network. Emotion, 8 (10), 355-358. 